\title{
A Survey on Motion Detection in a Video
}

\author{
Mandeep Kaur ${ }^{1}$, Pratibha Goyal ${ }^{2}$ \\ Student, Computer and Science Engineering, DBU, Khanna, India ${ }^{1}$ \\ Assistant Professor, Computer and Science Engineering, DBU, Khanna, India ${ }^{2}$
}

\begin{abstract}
Motion Detection is a process of detecting a change in the position of the object relative to its environment or vice-versa. As time changes computer applications are becoming important part in every field. Due to which it has been used in several applications. The verification of the object from a video and then tracking of that object is an significant task in computer vision. Object is a thing of interest whose movement is to record. It can be anything of any dimensions. Video is a recording of moving visual images and to record the movements of the object in the video is quite a complex task. Object detection is termed as to detect or locate objects from consecutive frames of a video file. For object detection, object representation is required. There are many techniques that came into existence to capture the motions of the object but every technique is having their own merits and demerits. One of the merit is that they capture every motion of the object in the video while demerit contains the concept of Computational Cost, Accuracy, Time, Noise, Shadow Effect, only Major Movement Detection, Detection of Stationary Objects ,etc. Also most of the cameras produce a noisy image, which result into a motion in that such places, where there is no motion at all. This paper presents a survey of different motion detection techniques. This survey paper includes background subtraction method, temporal differencing, statistical approach, and optical flow method and then comparison is made on them, with this a feature extraction algorithm can be used to examine the difference in the frames and thus, object can be detected in it. The purpose of a feature extraction is to obtain descriptive quantities (description) and reduce the dimensionality of data without losing relevant information. The dimensionality of a data defines to the number of values (i.e. dimensions) of a single measurement. A visual feature refers here are: - colour, texture and shape, etc.
\end{abstract}

Keywords: Video Tracking; Motion Detection,Feature Extraction,Dimensionality.

\section{INTRODUCTION}

A single still image provides a snapshot of a scene, the different frames of a video taken over time registers the dynamics (movement) in the scene, making it possible to capture motion in the sequence. Object detection in videos involves the detection of an object in a sequence of image frames. On the other hand, tracking is a process of detecting the moving multiple interested objects in a video file or camera depend upon the needs. In technical term, object tracking defined as the route or path of an interested or required object in the frame plane which is moving around the image plane. It is becoming the most emerging technology of nowadays due to the computational power, good quality and low cost video camera.
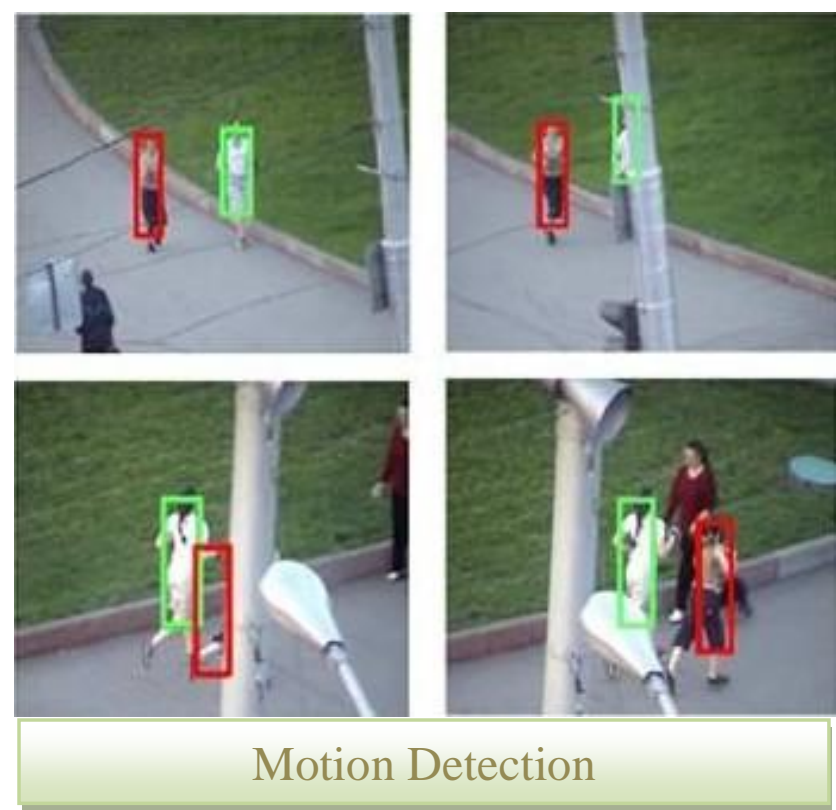
As the need of automated video system are implanting users are showing interest in object tracking algorithm. Video cameras are the most commonly used sensors in variety of computer applications. In video processing, a video can be presented with some hierarchical structure units, for as scene, shot and a frame. Also, video frame is the first lowest level in the hierarchical structure. In video retrieval, a video sequence must be first divided into a given number of video shots. A video shot can be termed as an image or video frame sequence that represents continuous action occurring while moving frames into a sequence. The frames in a video shot can be recorded from a single operation of a camera. The resulted video sequence consists of two or more video shots. The videos are made up of successive images (frames) which move fast enough so, that human eye can realize them as a continuous shot. Now, for any processing on video we need to the frame.

The video analysis is done by following three steps:

1. Detection of the required object that is moving.

2. Tracking of the selective object into respective frames.

3. Analysis of the path of the object.

Thus movement occurs between the objects is driven by the movement occurs between the camera and the watched scene. However, it is difficult to access the moving object's route or path in the video frame plane. Object tracking has been used. A typical strategy may used for tracking is to first segment a frame into a number of regions based on visual features like color and texture, subsequently merging of regions with similar motion vectors can be performed subject to certain constraints such as spatial neighbourhood of the pixels.

\section{OBJECT DETECTION TECHNIQUES}

\section{A. Point Detectors}

We are mostly interested in detecting point features in an image. These features are usually defined as regions in the image where there is significant edge strength in two or more directions. These are vital in searching points from the video frame having a surface in a particular area. Merit of using this type of detectors is that an interesting or concerned point remains unchanged even though enlightenment and camera perspective remains changing. Some of the point detectors used in are Harris detector, Moravec's detector, KLT detector etc.

\section{B. Temporal Differencing Method}

Temporal method computes the difference between the two consecutive frames after thresholding, resultant is the motion detection between the two frames (images).For this to achieve, subtract the previous image from the present frame. If the pixel difference is more than the set threshold value $\mathrm{T}$, this indicates that the pixels occur within the moving object; otherwise, they are the part of background pixels. The moving object can be detected after applying threshold operation. This method does not detect stationary objects but these are very sensitive to Threshold limit. Temporal Differencing method is highly adaptive to the changes in the scene as most recent frames are involved in the calculation of the moving regions.

\section{Background Subtraction Method}

The background subtraction method is the most common method of motion detection. In this type of detection, a scene is representing through establishment display and then performs deviations from model from one to every another approaching frame. In other words, it uses the difference of the current image and the background image and then if any change occurs in a picture results into change in an object or moving object. Thus pixels contributed to the region and acquire the variations in the frame for more processing. Here we consider the first frame as the background frame and then that frame is subtracted from current frame to detect moving object. Background subtraction method is used in Hidden Markov Models, frame differencing region based etc. Background subtraction method has three approaches and that are:-foreground region detection, background maintenance and post processing.

\section{Statical approach}

In this the whole picture is divided into comparable areas called segments. Thus it involves two parts i.e. in the first part criteria that will be used for the allotment of the segment and second part will be used to make strategy to attain useful division between the segments. This approach is becoming more popular due to its reliability in scenes that contain noise, illumination changes and shadows.

\section{E. Optical Flow}

The basic idea is, to calculate the image optical flow field, and do clustering processing according to the optical flow distribution features of image. This method gives the complete motion details and detects the moving object from the 
background better, due to a large quantity of calculations, sensitivity to noise and poor anti-noise performance; make this method not suitable for real-time demanding occasions. Optical Flow method can detect the motion in the video even from a movable camera and with moving background, but the optical flow methods are quite computationally complex and are not able to be used in real-time environment without specialized hardware.

\section{CHALlengeS}

There are some difficultis that are being encountered during detection and tracking of the motion of the object and they are :-
A. Occlusion(partial/full)
B. Environment (Challenging Weather)
C. Camera Movement
D. Object's Motion Speed
E. Cameras produce a Noisey image
F. Complex Object Behavoiur
G. Illumination
H. Complex shape of objects
I. Control Applications(Head Tracking for Video Conferencing)
J. Sometimes Bootstraping Strategy is to be applied

\section{APPLICATIONS OF MOTION DETECTORS AND TRACKING}
A. Video Surveillance
B. Traffic Monitoring Applications
C. Medical Analysis
D. Robotics(Human-Computer Interection)
E. Video Indexing
F. Vehicle Navigation(Automatic Guidance)
G. Motion based Recognition(Gesture Recognition)
H. Video saving
I. Motion Alarms
J. Control Applications(Head Tracking for Video Conferencing)

\section{FEATURE TYPES}

Motion Detection uses feature type techniques to recognize any moving object. A feature is a fascinating part of an image, like as a corner, blob, edge, or line on that basis moving object is detected i.e. motion is detected.

- LBP: It is a feature extraction technique and it has its excellence in Classification, clustering \& segmentation process. Its features can be used as small patterns, which are balanced or regular with reference to the monotonic grey scale revolution.

- POC: Phase Correlation, is one of the image matching technique that has been applied to the biometric authentication and computer vision problems. The height and position value of the correlation peak depicts difference between the two images.

- Haar Classifier: Haar Like features are composed of two or three jointed black and white rectangles. The integral image is defined as the sum total of the pixel values of the original image. The value at any location (say(x, y) coordinates) of the integral image is the summation of the image pixels above and to the left of location (x, y). The integral image value can be calculated by discovering the difference between the summations of pixel gray level values within the black and white rectangular regions compared with raw pixel values.

- AdaBoost: This algorithm is used to weight the choosen weak classifier. All the weak classifiers are classified to many cascades with the help of optimization process. Within each stage, a group of several weak classifiers are drill using this algorithm.

- Gabor Wavelet: In this algorithm, the images which are represented by Gabor wavelets algorithm are selected for its biological matter and technical properties. The Gabor wavelets are of almost similar structure (appearance) as the receptive fields of simple cells in the primary visual cortex. We use Gabor wavelets for image representation since it depicts the image based on the way the human mind does. This makes modeling computer vision based on human vision a more systematic and powerful process. 


\section{CONCLUSIONS}

In this survey paper all the main terminologies of object detection have been included. These include object detection methods and feature selection .Most commonly used and well recognized methods for these phases have been explained in details. Different methods for object detection are like Point Detectors, frame difference, optical flow and background subtraction. Most commonly used method is back- ground subtraction.

In the video object tracking system the main focus till date is to check the frames and by the use of Euclidean distance approaches which make use of the differences between the previous frames along with the present frames. This technique is successful in most of cases but it is very simple approach which can be non-effective in most of cases and the chances to get the perfect object detection in the live frames. So there is requirement of the algorithm which will be more productive this can be done by withdrawing the feature of the image( or can say the frames).Among the many methods of object classification mostly researchers give preference to the texture based and color based object classification techniques. Advance study may open the paths to find efficient algorithms to reduce computational cost and to decrease the time required for detecting the object for variety of videos containing different characteristics and to increase accuracy rate. Also, most of the techniques were not able to detect the minor movement of the object. So, linear binary pattern technique can used for the extraction and matching of the feature. This technique will help in the detection of the minor movements of the object. LBP is considered as one of the efficient technique for the feature extraction.

\section{ACKNOWLEDGEMENT}

First and foremost I thank Almighty God for all the blessings endowed on me. I would also like to express my immense pleasure and gratitude towards my guide Mrs. Pratibha Goyal assistant professor of Computer and science department, DBU. I am also very thankful to my teacher Ms. Jyoti Arora for her whole hearted support .I deeply express my sincere thanks to Khushboo mam, HOD of Computer Science And Engineering department of Desh Bhagat University. I would like to extend my gratitude to all other faculty members for their help advice.

\section{REFERENCES}

[1] Prasad K, Sharma Richa and Wadhwani D “A Review On Object Detection In Video Processing” in the International Journal of Science and Technology,Vol. 5, Pp.454-463, December, 2012.

[2] Shantaiya S, Verma K and Mehta K," Study and Analysis of Methods of Object Detection in Video", International Journal of Soft Computing and Engineering (IJSCE), ISSN: 2231-2307, Vol. 2, Pp. 343-352, Issue-6, January 2013.

[3] K Amaleswarao , G Vijayadeep and U Shivaji, "Improved Background Matching Framework For Motion Detection" in the International Journal of Computer Trends and Technology, Vol. 4, Pp. 243-252, Issue-8, August,2013.

[4] Hussain M A and Kharat G U , "Robust Human Motion Detection and Tracking In Dynamic Background", International Journal of Soft Computing and Engineering (IJSCE) ISSN: 2231-2307, Vol. 2, Pp. 547-556, Issue-6, January 2013

[5] M.Usha," Motion detection in compressed video using macro block classification”, ACIJ, Vol.5, No.2/3,Pp 1-15, 2014.

[6] Rakibe R S," Human Motion Detection using Background Subtraction Algorithm”, IJARCSSE, Vol. 4, Issue 2, Pp. 343-351, February 2014.

[7] Rajput P," New Trends in Object Motion Analysis", ijarcsse, Vol. 4, Issue 4, Pp 185- 189,2014.

[8] Deepika T,"Motion detection in real time video surveillance with movement frame capture and auto record", International Journal of Innovative Research in Science, Engineering and Technology, 2014

[9] Kuriakose I M , "Survey on Human Motion Detection In Static Background" American Journal of Engineering Research (AJER), Vol. 03, pp144-146,2014 Shantaiya S, Verma K and Mehta K," Study and Analysis of Methods of Object Detection in Video", International Journal of Soft Computing and Engineering (IJSCE), ISSN: 2231-2307, Vol. 2, Pp. 343-352, Issue-6, January 2013.

[10] Jadhav J J,"Moving Object Detection and Tracking for Video Survelliance", International Journal of Engineering Research and General Science, Vol. 2, Issue 4, June- July, 2014.

[11] Abdelali H A, Essannouni F, Essannouni L and Aboutajdine D, "Algorithm for Moving Object Detection and Tracking in Video Sequence Using Color Feature", IEEE, 2014.

[12] Patil S," Suspicious movement detection and tracking based on color histogram", IEEE, Pp 1-6, 2015.Rakibe R S," Human Motion Detection using Background Subtraction Algorithm", IJARCSSE, Vol. 4, Issue 2, Pp. 343-351, February 2014.

[13] Jadhav J and Patil Y," Moving Object Detection for Video Surveillance System”, International Journal of Computer Applications (0975 8887), National Conference on Emerging Trends in Advanced Communication Technologies (NCETACT- 2015).

[14] Sukanya C M, Gokul R and Paul V," A Survey on Object Recognition Methods", IJCSET jan 2016.

[15] Wady S H and Ahmed H O," Ethnicity Identification based on Fusion Strategy of Local and Global Features Extraction" International Journal of Multidisciplinary and Current research, Vol.4 (March/April 2016) . 